\title{
HANDY MICROSCOPIC CLOSE-RANGE VIDEOGRAMMETRY
}

\author{
F. Esmaeili ${ }^{\text {a, } *, \text { H. Ebadi }}{ }^{b}$ \\ ${ }^{a}$ Geodesy and Geomatics Eng. Faculty, K.N.Toosi University of Technology, Tehran, Iran - faridesm@mail.kntu.ac.ir \\ ${ }^{\mathrm{b}}$ Geodesy and Geomatics Eng. Faculty, K.N.Toosi University of Technology, Tehran, Iran - ebadi@kntu.ac.ir
}

KEY WORDS: Close-range photogrammetry, Microscopic Imaging, Micrometer Calibration Ruler, Videogrammetry, Hand USB Digital Microscope

\begin{abstract}
:
The modeling of small-scale objects is used in different applications such as medicine, industry, and cultural heritage. The capability of modeling small-scale objects using imaging with the help of hand USB digital microscopes and use of videogrammetry techniques has been implemented and evaluated in this paper. Use of this equipment and convergent imaging of the environment for modeling, provides an appropriate set of images for generation of three-dimensional models. The results of the measurements made with the help of a microscope micrometer calibration ruler have demonstrated that self-calibration of a hand camera-microscope set can help obtain a three-dimensional detail extraction precision of about 0.1 millimeters on small-scale environments.
\end{abstract}

\section{INTRODUCTION}

Nowadays, Close-range photogrammetry is used in different fields as an appropriate measurement tool. In some applications, however, close-range photogrammetry can be used as the only effective tool for measurement and geometric modeling of the measured object in view of the nature of the object. One of these fields concerns measurement of very small objects. It is a challenge to measure small objects with dimensions below 1 centimeter to even 0.01 millimeters using this method. In 2010, Yanagi provided an evaluation of small object modeling using close-range photogrammetry method with the help of macro-lens cameras (H. Yanagi, et al., 2010). They obtained a precision of 0.05 millimetres in modeling the surface of a coin and displaying its details geometrically. In 2012, Gun used basic microscopic photogrammetry for precise measurement of the length of a scale bar (Gan, et al.,2012). In 2016, Gontard applied photogrammetry in a scanning electron microscope (SEM) to study the three-dimensional shape and surface texture of a nano scale LiTi2(PO4)3 particle (Gontard, et al., 2016).

Niederöst and his colleagues modelled small objects with dimensions of about 10 micrometers using microscopic imaging and applied the Shape From Focus method (Niederöst, et al.,2005). In order to make it possible to image objects from all sides at appropriate angles, they used a circular platform with the capability of changing the angle with respect to the microscope lens. Modeling minute objects has different applications particularly in medical fields. These include detection of the growth rate and measurement of tumours, mycology and parasitology, and detection of skin and eye diseases. Furthermore, applications of quality control in the industrial fields, examination of different materials in the fields of chemistry and metallurgy, and applications of cultural heritage also make use of measurement and modeling of smallscale objects using close-range photogrammetry method in different cases (Carli, L., 2010). In some of the applications, use of ordinary cameras with great magnitude and use of macro lenses can sometimes provide proper images from minute objects as photogrammetric observations up to about 0.1 millimetres. But when smaller measurements are needed, however, digital cameras installed on microscopes are often utilized. One of the problems involved in using large electronic microscopes is the impossibility to change their imaging angles with respect to the object. In such cases, the position of the object with respect to the microscope is often changed, so that images can be obtained at proper angles from all sides of the object. Furthermore, the disadvantages of these microscopes include their high prices and the impossibility to move them easily. Use of portable, inexpensive digital microscopes for obtaining photogrammetry-based data with the video-based methods for modeling minute objects has been investigated in this paper. The wide magnitude range of this type of equipment and the increase in the quality of the obtained images through employment of new imaging technologies has made it possible to model a wide range of small-scale objects with the help of handy microscopes. Below, the equipment used in this paper will first be introduced. Next, the stages of implementation and results will be examined and evaluated.

\section{METHODOLOGY AND EXPERIMENTAL RESULTS}

Despite their high magnitudes, desktop electronic microscopes don't have the capability to be moved or to obtain convergent images from the environment for modeling. For imaging all the components of the environment, peripheral equipment, such as circular platforms with the capability of changing the angle with respect to the horizon, is used. Handy microscopes with digital cameras have been in use during recent years for many medical and industrial applications (Figure 1). Their capability of easy movement makes it possible to obtain images at proper intersection angles with respect to the object.

\footnotetext{
* Corresponding author
} 


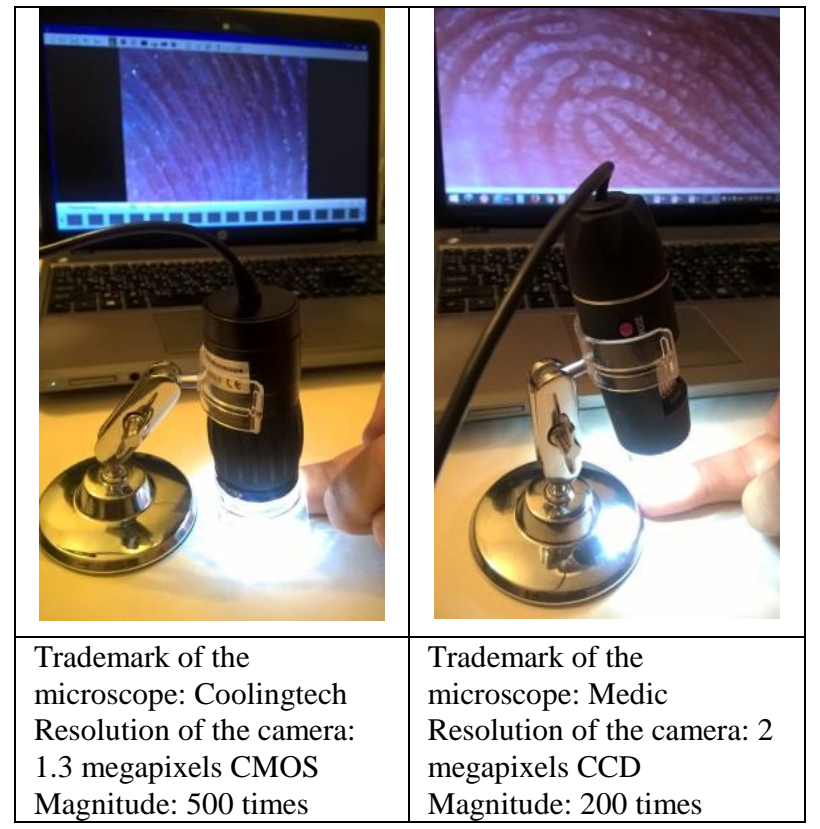

Figure 1. Hand microscopes used in the research and their specifications

In handy microscopes, imaging the environment sometimes involves shakes even if the microscope is located on the base, which leads to a decrease in the quality of the obtained images. In this paper, the observations to be obtained for modeling based on imaging at a rate of $7.5 \mathrm{f} / \mathrm{s}$ from the relevant parts of the object were suggested. One of the major challenges in microscopic imaging is different focuses in the field of view based on different depths (Figure 2). Obtaining videos from the environment with handy microscopes makes it possible to obtain clear frames from all parts of the object in light of the constant change in the distance between the camera and the object, and proper observations can finally be provided for modeling different parts through combination of the images.

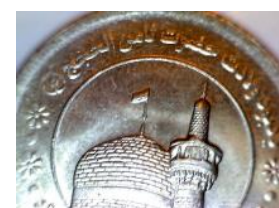

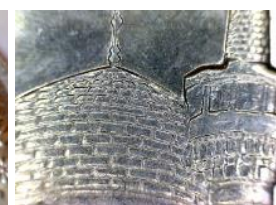

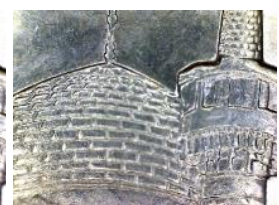

Figure 2. Different focuses in different parts of the surface of a coin. a) View of the coin. b and c) Different focuses on the coin

We are often confronted with virtual parameters in calibration of the cameras located on microscopes. There are no fixed parameters for calibration of such cameras due to combination of the microscope lenses in the imaging process. Therefore, the set of interior orientation parameters for the camera can be estimated in each project with an appropriate number of images using the self-calibration method while the distances between the lenses are constant.

One of the most frequent methods for making real measurements in microscopic imaging is the use of microscope micrometre calibration rulers (Celestron, 2017) (Figure 3-a). Different marks of particular dimensions were printed in these calibration rulers on a small-scale slide. For obtaining the actual lengths in photographic measurements, the checkmarks available on the image can be measured with the help of a calibration ruler at a fixed focal length used for measurements as shown in Figure 3-b, and the checkmarks can then help make the measurements also on the original images. The measurement can also be made at the same time as the microscope micrometre calibration ruler is located on the environment (Figure 4).
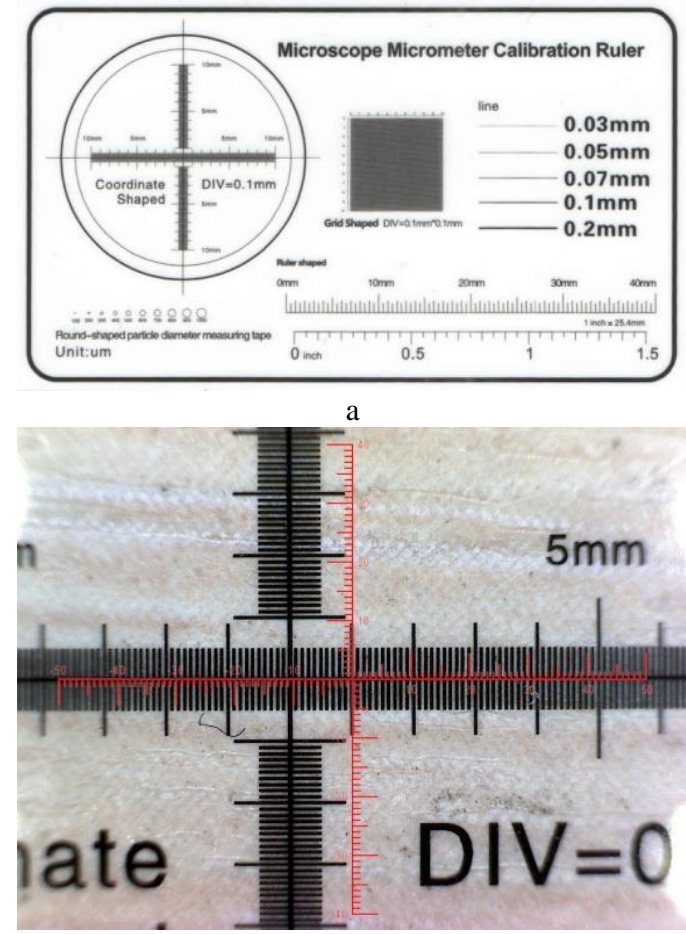

b

Figure 3. a) A microscope micrometer calibration ruler b) Measurement of the image checkmarks with the help of a calibration ruler
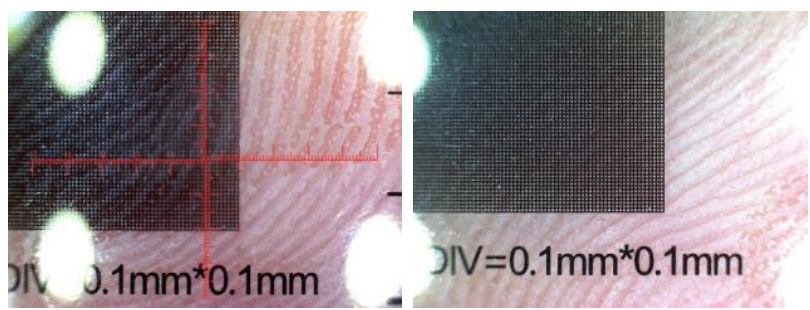

Figure 4. Direct measurement with the help of a microscope micrometer calibration ruler on the environment

For evaluation of the performance of microscopes equipped with digital cameras, two types of this equipment (Figure 1) have been used in this research for modeling two different objects with different magnitudes (Figures 5-a and 5-b). The first environment is a human index fingerprint, and the second is the great toenail surface. The imaging has been performed in the form of a convergent network with respect to the environment. 88 frames from the video were used for modeling the object. The results of the model generated from the objects are displayed in Figure 6. 


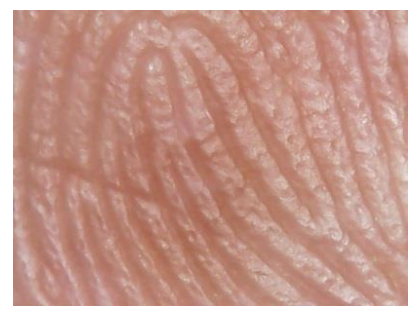

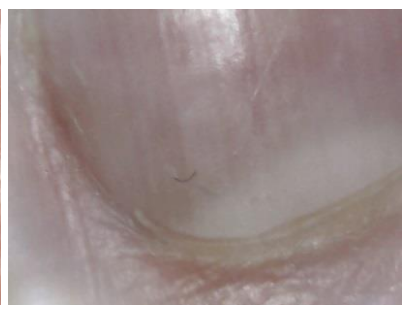

Figure 5. Sample microscopic images obtained for modeling. a) Fingerprint. b) Toenail surface

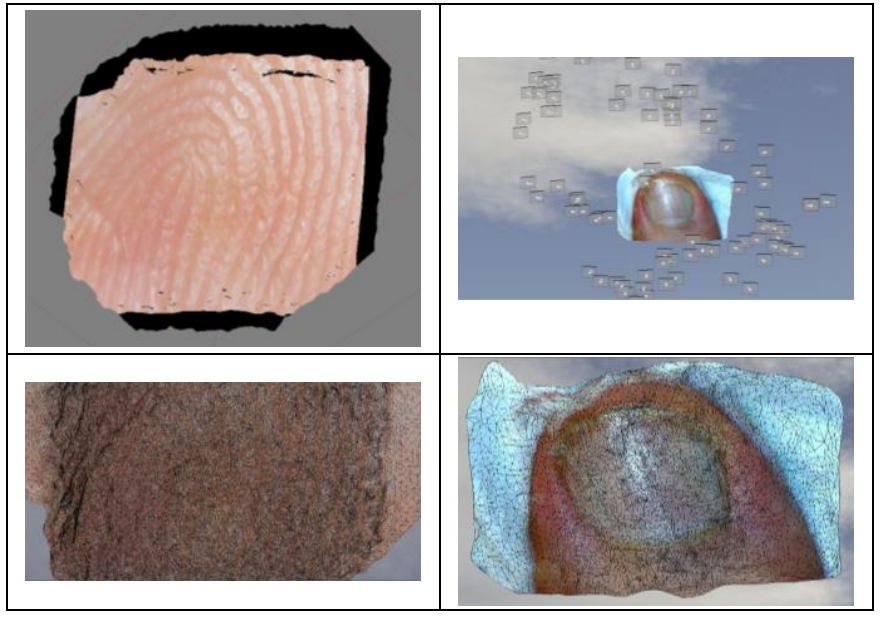

Figure 6. Textured three-dimensional models generated from the objects under investigation. a) Fingerprint. b) Toenail surface

As observed in the results of the performed modeling, an appropriate density of points with respect to the environment dimensions has been generated along with plenty of details. Extraction of textured three-dimensional models with these details from small-scale objects constitutes one of the unique capabilities of photogrammetry-based methods. Two types of microscopes with different camera resolutions and different magnitudes have been used in this project. The Medic microscope has a more convex lens than the Coolingtech microscope. Therefore, focal length difference is higher in the central parts and around in the Medic images than in the Coolingtech images. Despite their low resolution, the Coolingtech microscope images have greater magnitudes, and generate more resonant images. Based on the measurements made with the help of the microscope micrometer calibration ruler, the details on the environment have been modelled with an average precision of about 0.1 millimetres. The results demonstrate that hand microscopes equipped with inexpensive digital cameras can be used extensively for different applications of modeling small-scale objects.

\section{CONCLUSIONS}

The capability of handy microscopes equipped with online imaging using videogrammetry method to produce threedimensional modeling of small-scale objects was implemented and evaluated in this paper. Use of video images eliminated the problem of lack of a single focus in all the environment components in microscopic images to a large extent.
Furthermore, image shakes and hazes were observed less frequently in obtained video images than in photography. Use of handy microscopes makes it possible to obtain convergent images at different angles with respect to the environment. It is also possible to use self-calibration method for estimation of the interior orientation parameters to obtain high precisions. To sum up, this technology can be used for modeling and specifying the coordinates of minute object details with a precision of about 0.1 millimetres.

\section{REFERENCES}

Carli, L., 2010. 3D-SEM metrology for coordinate measurements at the nanometer scale. Department of Mechanical Engineering, Technical University of Denmark. $\mathrm{PhD}$ Thesis.

Celestron, 2017. Celestron Handheld Digital Microscope Description,http://www.celestron.com/browseshop/microscopes/digital-microscopes/handheld-digitalmicroscope-pro (15 April 2017)

H. Yanagi and H. Chikatsu. 2010. 3d modeling of small objects using macro lens in digital very close range photogrammetry, in International Archives of Photogrammetry, Remote Sensing and Spatial Information Sciences, Newcastle upon Tyne.

Lionel C Gontard, Roland Schierholz, Shicheng Yu, Jesús Cintas, and Rafal E Dunin-Borkowski, 2016. Photogrammetry of the three-dimensional shape and texture of a nanoscale particle using scanning electron microscopy and freeware software. July 2016, Ultramicroscopy.

Markus Niederöst, Jana Niederöst and Jiř́ Ščučka, 2005. Automatic $3 \mathrm{~d}$ reconstruction and visualization of microscopic objects from a monoscopic multifocus image sequence, in International Archives of the Photogrammetry, Remote Sensing and Spatial Information Sciences.

Xiao-chuan Gan, Ming-zhao He, Lian-fu Li, Xiao-you Ye, and Jian-shuang Li, 2012. Photogrammetric scale-bar measurement method based on microscopic image aiming, in 6th International Symposium on Advanced Optical Manufacturing and Testing Technologies: Optical Test and Measurement Technology and Equipment. 